\title{
Prognostic importance of DNA flow cytometry in non-Hodgkin's lymphomas
}

\author{
JC MACARTNEY,* RS CAMPLEJOHN, $\dagger$ JULIE ALDER, $\dagger$ MG STONE, $\dagger$ \\ GILL POWELL*
}

From the *Department of Histopathology, and the †Richard Dimbleby Department of Cancer Research, Rayne Institute, St Thomas's Hospital Medical School, London

SUMMARY Eighty one cases of non-Hodgkin's lymphoma were examined by DNA flow cytometry, using fixed embedded histological tissue. The frequency of detection of DNA aneuploidy and the values for $\mathbf{S}$ phase fractions depended on the histological subtype and grade of lymphoma. Twenty two of the patients with low grade centroblastic/centrocytic non-Hodgkin's lymphoma had repeat biopsies. Eleven of these patients remained histologically and cytometrically stable, but the remaining eleven transformed into high grade non-Hodgkin's lymphoma. The mean value for the $S$ phase fraction in the initial biopsy specimens from patients which transformed was higher than that for patients whose lymphomas remained stable $(\mathrm{p}<0.001)$. It is proposed that estimates of $S$ phase fraction prospectively identify patients with low grade non-Hodgkin's lymphoma at risk from transformation.

Nun-Hodgkin's lymphomas are a heterogenous group of tumours. This is reflected in the existence of several classifications based on cytological, immunological, and prognostic features ${ }^{1-4}$ Grade, stage, and response to treatment are also important prognostic factors. ${ }^{5-7}$ Cell kinetic variables hae been measured in an attempt to define the correlation between the proliferative activity of these tumours and their biological aggressiveness. ${ }^{8-20}$ The proportion of tumour cells synthesising DNA (labelling index or $\mathbf{S}$ phase fraction) has been measured directly in vitro by ${ }^{3} \mathrm{H}$-thymidine incorporation, or DNA flow cytometry, and indirectly, using immunocytochemical methods to detect the expression of markers identifying the growth fraction. Although these studies indicated overall kinetic heterogeneity of nonHodgkin's lymphoma, it is generally agreed that high grade tumours are more likely to possess abnormal amounts of DNA (aneuploidy) and to show higher proportions of cells synthesising DNA or expressing markers such as $\mathrm{K} 1-67$ and transferrin receptor. High labelling indices or $\mathrm{S}$ phase fractions have also been associated with a lower rate of remission induction and duration of remission. ${ }^{21} 22$

We recently confirmed that DNA flow cytometry on histologically processed lymph node biopsy material compared favourably with results obtained from fresh tissue. ${ }^{23}$ In this study we applied this technique

Accepted for publication 16 January 1986 to a larger group of non-Hodgkin's lymphoma. The principal aim was to assess whether cytometric data could be used prognostically to identify which low grade follicle centre cell non-Hodgkin's lymphomas were more likely to transform into a high grade, with a more aggressive clinical course. ${ }^{624}$

\section{Material and methods}

DNA flow cytometry was performed on cell suspensions prepared from formalin fixed paraffin embedded blocks of human non-Hodgkin's lymphoma. Eighty one cases with non-Hodgkin's lymphoma were examined using the method described by Hedley et al, ${ }^{25}$ with minor modifications. ${ }^{23}$

\section{TISSUES}

Histological material was selected from the files of the department of pathology, at this hospital. No follow up data were included in the study as information was not always available. Moreover, uniform staging procedures and therapeutic schedules were not used for the patients. A total of 131 separate biopsy samples from the 81 patients were studied. Each tumour was classified and graded using Kiel nomenclature. ${ }^{3}$ In 65 of the patients the histological diagnosis was supplemented by immunohistochemical examination for lymphocyte markers. Cases classified as low grade centroblastic/centrocytic non-Hodgkin's lymphoma with follicular growth patterns excluded those with 
tumours composed predominantly of large follicle centre cells.

\section{CELL SUSPENSIONS}

Representative blocks were selected from every biopsy specimen and $30 \mu \mathrm{m}$ sections cut. These sections were dewaxed in xylene and rehydrated through a series of alcohols $(100 \%, 95 \%, 70 \%$, and $50 \%)$ into distilled water. The sections were then treated for 30 minutes at $37^{\circ} \mathrm{C}$ in a $5 \mathrm{mg} / \mathrm{ml}$ solution of pepsin (Sigma, Dorset, England) at pH 1.5. The resulting suspension was spun at $2000 \mathrm{rpm}$ for three minutes and resuspended in $5 \mathrm{ml}$ of Isoton (Coulter Electronics, Luton, England) containing $1 \mu \mathrm{g} / \mathrm{ml}$ DAPI (4' 6-diamidino-2 phenylindol-hydrochloride; Boehringer, West Germany). The suspensions were passed through a 25 gauge needle before filtration through 35 $\mu \mathrm{m}$ pore nylon gauze.

\section{FLOW CYTOMETRY}

Samples were analysed as before with a Becton Dickinson FACS Analyzer. ${ }^{23}$ To construct each histogram 10000-15000 cells were scanned, and the results were stored on a disc for further study. The DNA index was determined and, where possible, the $S$ phase fractions calculated by the method of Baisch et $a l .{ }^{26}$ The DNA index is calculated by measuring the position of any aneuploid peak relative to the normal $G_{0} / G_{1}$ peak. A DNA index of 1.0 indicates only the presence of diploid cells. DNA aneuploidy was documented only if there was clear evidence of a second $G_{0} / G_{1}$ peak on the flow cytometric tracing. Reference samples, such as chicken red blood cells which are used to calibrate the $G_{0} / G_{1}$ diploid peak, were not used in this study as they fail to give reproducible results with fixed tissue. ${ }^{25}$ Where there was evidence of DNA aneuploidy, the $S$ phase fraction was calculated using the average mid $S$ phase height according to the method described by Frankfurt et al. ${ }^{27}$ This

Classification and ploidy of lymphomas (Figures in parentheses are numbers \%)

\begin{tabular}{lcc}
\hline Histology* & No of cases & DNA aneuploidy \\
\hline Low grade & & \\
Malignant lymphoma & 11 & $0(0)$ \\
$\quad$ lymphocytic & 7 & $3(43)$ \\
Lymphoplasmacytoid & 34 & $4(12)$ \\
Centroblastic/centrocytic $†$ & 10 & $7(70)$ \\
High grade & 8 & $5(63)$ \\
Centroblastic & 7 & $3(43)$ \\
Lymphoblastic & 4 & $3(75)$ \\
Pleomorphic T cell & 81 & $25(31)$ \\
Unclassified & & \\
Total & &
\end{tabular}

*Initial biopsy;

$\dagger$ A further three of 11 cases became aneuploid among the group which transformed to high grade lymphoma;

Excludes the 11 cases of centroblastic and centrocytic lymphomas which transformed to high grade tumours. method applies only when there are substantial flat zones for both of the $S$ phase regions on the plot. It is a prerequisite that these zones are not overlapped by corresponding $G_{0} / G_{1}$ or $G 2 / M$ peaks, although this was not applied to any tumours exhibiting multiple DNA aneuploid peaks. A full peak coefficient of variation for the $G_{0} / G_{1}$ peak was calculated for each sample using software supplied by Becton Dickinson.

\section{Results}

\section{HISTOLOGY}

The Table shows the histological classification of the 81 patients at presentation. Four cases could not be classified; 34 had two or more biopsies. These include 22 patients classified at presentation as low grade follicle centre cell non-Hodgkin's lymphoma of centroblastic/centrocytic subtype with a follicular growth pattern. Eleven (group A) of these maintained a low grade histological appearance with a mean interval between biopsies of 3.7 years. The other group (group B) (11 cases) subsequently developed high grade centroblastic non-Hodgkin's lymphoma with a mean interval of 3.4 years between initial diagnosis of low grade non-Hodgkin's lymphoma and evidence of transformation. In a further 12 low grade centroblastic/centrocytic cases either only a single biopsy specimen had been taken or the interval between first and second biopsy had been less than six months. The remaining 12 of 34 patients who had had more than one biopsy provided specimens of heterogeneous histological subtype.

\section{PLOIDY}

Overall, 28 of the 81 patients in this series had DNA aneuploid tracings. In eight of these cases multiple aneuploid lines occurred, and 10 showed changes in the pattern of DNA index with time. This was usually. a change from a diploid to an aneuploid tracing. The modal value for tumours with an aneuploid DNA index in the series was 1.1 with a second minor peak at 1.8-1.9. The relation of DNA aneuploidy to histological diagnosis is shown in the Table.

No evidence of DNA aneuploidy was found in low grade non-Hodgkin's lymphoma classified as malignant lymphoma, lymphocytic, and the number of cases showing DNA aneuploidy was also low in low grade centroblastic/centrocytic non-Hodgkin's lymphoma. There was no significant difference in the incidence of DNA aneuploidy observed between the two subgroups (group A one case, group B two cases) of centroblastic/centrocytic non-Hodgkin's lymphoma. The only apparent exception to the rare occurrence of DNA aneuploidy in low grade non-Hodgkin's lymphoma was the presence of aneuploidy in three of seven cases of lymphoplasmacytoid non-Hodgkin's 
lymphoma, although the numbers in this category were small. A fourth patient became aneuploid, but only after undergoing transformation to a high grade non-Hodgkin's lymphoma.

In contrast, cases of high grade non-Hodgkin's lymphoma generally showed a much higher rate of DNA aneuploidy (Table). Cases classified as pleomorphic $\mathrm{T}$ cell lymphoma seemed to show an overall lower rate of DNA aneuploidy than other cases of high grade non-Hodgkin's lymphoma, although the numbers are again small. There was no correlation of flow cytometry results with lymphocyte marker studies, although these studies did not include transferrin receptor analysis.

\section{S PHASE FRACTION}

Fig. 1 shows the mean and scatter values of $S$ phase fractions for those cases in this series in which it was possible to measure this variable. In general, low grade non-Hodgkin's lymphoma had lower $\mathbf{S}$ phase fractions and, by comparison, high grade nonHodgkin's lymphoma had higher $S$ phase fractions. The scatter of values, however, was noticeably greater for cases of low grade centroblastic/centrocytic (mean $\% S=4.3$ (SE 0.3)) and lymphoplasmacytoid non-

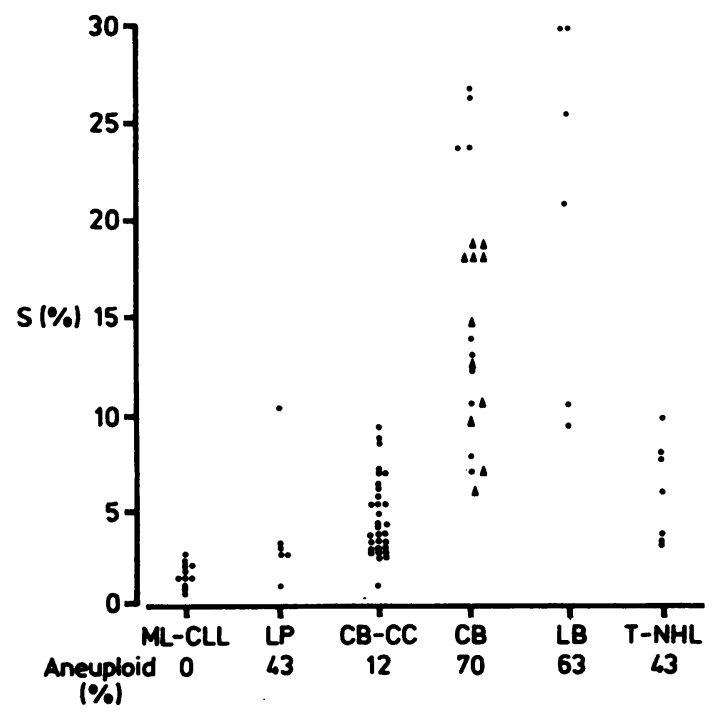

Fig. 1 S phase fractions for non-Hodgkin's lymphomas subtypes.

Closed circle denotes primary biopsy; triangle denotes secondary centroblastic lymphoma; $M L / C L L=$ malignant lymphoma lymphocytic; $L P=$ lymphoplasmacytoid non-Hodgkin's lymphoma; $C B / C C=$ centroblastic/ centrocytic non-Hodgkin's lymphoma; $C B=$ centroblastic non-Hodgkin's lymphoma; $L B=$ lymphoblastic non-Hodgkin's lymphoma; $T / N H L=$ pleomorphic $T$ cell non-Hodgkin's lymphoma.

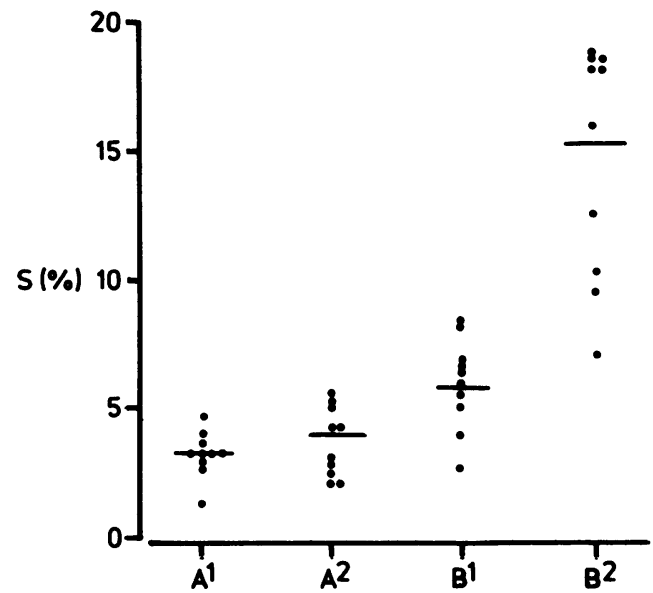

Fig. 2 phase fractions for two groups of low grade centroblastic/centrocytic non-Hodgkin's lymphomas ( $A$ and $B$ ). Bar denotes mean value for $S$ phase fraction. $A^{1}$ and $B^{1}$ are values from first biopsy samples. $A^{2}$ and $B^{2}$ are values from second biopsy samples.

Hodgkin's lymphoma when compared with that for low grade lymphocytic non-Hodgkin's lymphoma (mean \% S $=1.6(0.3)$ ). Among cases of high grade lymphoma the scatter is even greater, and cases of high grade $T$ cell non-Hodgkin's lymphoma show considerable overlap with low grade non-Hodgkin's lymphoma. Nevertheless, $\mathbf{S}$ phase fractions for high grade follicle centre cell lymphomas (mean 14.3 $(1.5) \%$ ) and lymphoblastic lymphomas (mean 21.4 (4)\%) were significantly higher than values for low grade lymphomas.

\section{PROGNOSTIC EFFECT OF S PHASE FRACTION}

In the 12 patients with mixed histology in whom repeat biopsies showed no change in the histology from the initial biopsy, other than those referred to below, measurements of $S$ phase fraction did not differ appreciably between the first and second sample, although there was a slight upward trend.

Fig. 2 shows the $S$ phase fractions in paired biopsy specimens from the two groups of patients with low grade centroblastic/centrocytic follicular nonHodgkin's lymphoma in the first biopsy sample. There were 11 cases in each of these groups, but $S$ phase fractions could only be calculated for 10 cases in both instances due to the effects of DNA aneuploidy.

In group $\mathbf{A}$ the first and second biopsy specimens both showed low grade tumours with similar histological features. Although there was a slightly higher overall mean value for the $\mathbf{S}$ phase fractions in the second biopsies $(3.9(0.5) \%)$ compared with that of the first biopsies $(3.2(0.3) \%)$, these did not differ 
significantly from each other (paired $t$ test; $0.5>\mathrm{p}>$ $0 \cdot 1)$.

Fig. 2 also shows the paired values for $S$ phase fractions from group B. In these cases the first biopsy specimens showed low grade centroblastic/ centrocytic follicular non-Hodgkin's lymphoma, but the tumours subsequently transformed into large cell high grade non-Hodgkin's lymphoma. As expected, the $\mathrm{S}$ phase fractions in the second biopsies after transformation (mean $14.9(1.6) \%$ ) were much higher than those found in the first biopsies $6 \cdot 0(0 \cdot 6) \%, p<$ 0.001 ). In addition, however, the $S$ phase fractions of first biopsies from patients in group B were significantly higher than those for the primary biopsies from group $A .(t=4.5, p<0.001)$.

\section{COEFFICIENT OF VARIATION}

The mean full peak coefficient of variation for the $G_{0} / G_{1}$ peak on all biopsy specimens examined cytometrically in the series was 4.0 (range of 1.8 to 5.5 ). In general, histological material from specimens fixed in formalin gave rather better quality results than that for specimens fixed in formalin and acetic acid. Lymph node biopsy material fixed in Bouin's fixative was unsuitable for DNA flow cytometry.

\section{Discussion}

In a previous paper based on a very small number of patients we found only one case of DNA aneuploidy. ${ }^{23}$ In this larger series the overall incidence of DNA aneuploidy was $35 \%$. This was, however, clearly dependent on the subtype of nonHodgkin's lymphoma under consideration and its natural history. Thus no evidence of DNA aneuploidy was observed in the cases of malignant lymphoma, lymphocytic, whereas $12 \%$ of low grade centroblastic/centrocytic non-Hodgkin's lymphoma were aneuploid, and the percentage rose to $60-75 \%$ in various forms of high grade non-Hodgkin's lymphoma. Although these figures are comparable with certain other studies, they are considerably lower than those of Diamond et al ${ }^{17}$ and Braylan. ${ }^{18}$ Braylan obtained an overall prevalence of $80 \%$ for DNA aneuploidy, but this reflects the increased sensitivity afforded by restricting cytometric measurements to neoplastic lymphoid cells identified using surface markers. Conversely, a notably low incidence $(<10 \%)$ reported in another study ${ }^{10}$ was almost certainly due to the sampling problems associated with the technique of microdensitometry used to measure cellular DNA content. From published work it is also apparent that 1-2\% of cases will exhibit hypodiploidy. ${ }^{1418}$ Due to the absence of a DNA calibration value when using histologically processed material such case(s) will have been misclassified as hyperdiploid in our series. We think it unlikely, however, that DNA aneuploidy would be completely missed as we have yet to sample fresh human neoplastic tissue and fail to obtain a diploid peak in the presence of an aneuploid peak.

Thus the frequency of DNA aneuploidy depends on the mixture of cases studied and the techniques used to identify aneuploidy. Given that we and others have obtained a modal value of 1.1 for aneuploid DNA indices and that the flow cytometer is unlikely to be able to discriminate in the DNA index range of $1 \cdot 0-1 \cdot 1$ without very low values for the $G_{0} / G_{1}$ coefficient of variation, all published figures for aneuploidy in non-Hodgkin's lymphoma are likely to be minimum values.

The main finding of this study was the difference in the values for $\mathbf{S}$ phase fractions when cases of low grade non-Hodgkin's lymphoma, which ultimately transformed into high grade tumours, were compared with cases of low grade non-Hodgkin's lymphoma that remained stable. Clearly this difference might be exploited prospectively to identify patients who are at risk from developing transformed lymphomas and to select appropriate therapeutic schedules. Other workers have shown a correlation between $S$ phase fractions of non-Hodgkin's lymphoma and the induction of remission or the remission duration. ${ }^{21} 22$

The results reported here, however, differ in that the two groups compared in this study underwent a definite relapse but with different outcomes.

Before this prognostic difference can be used therapeutically, certain problems need to be solved. Our patients were all studied retrospectively and were not clinically or pathologically staged according to a standard protocol. The scatter of results from $S$ phase fractions that we obtained was quite high and depended on histological subtype. Similar variability has been observed in other reports of non-Hodgkin's lymphoma. ${ }^{8-20}$ For these reasons we have not defined an upper limit for $S$ phase fractions in low grade lymphomas, although by implication this could be set at about $8 \%$ for all low grade centroblastic/centrocytic lymphomas regardless of the natural history or proportion of small and large follicle centre cells (mean $+2 \mathrm{SD}$ ).

Before setting strict limits to $\mathrm{S}$ phase fractions, however, other factors influencing survival, such as the staging treatment and symptomatology of the patients, need to be controlled in future studies. The data on the effect of staging on measurements of $S$ phase fraction in non-Hodgkin's lymphoma are contradictory. Costa et al showed no correlation of labelling index to stage, using autoradiographic techniques, ${ }^{12}$ whereas others ${ }^{11}$ obtained lower labelling indices in more advanced stages. These results, ${ }^{11}$ however, should be viewed with caution, as scintil- 
lation counting was used and considerable doubts regarding the validity of measurements obtained have been expressed. ${ }^{28}$

It might be possible to overcome these deficiencies by studying a large number of patients and including other variables in the flow cytometric analysis, such as nuclear volume. Results of multivariable studies including volume measurements have been published but not in the same context as this study. ${ }^{13-15}$ Alternatively, cytometric measurements could be restricted to neoplastic lymphoid cells labelled with appropriate surface markers. This approach was adopted by Braylan $e t a^{18}$ but would necessitate a return to using fresh tissue samples, as our unpublished observations suggest that only the nuclei of fixed embedded tissue survive treatment with pepsin.

We thank Mrs Sheila King for typing the manuscript.

\section{References}

${ }^{1}$ Rappaport H. Tumors of the haemopoietic system. Atlas of tumour pathology. Washington DC: Armed Forces Institute of Pathology, 1966.

${ }^{2}$ Lukes RM, Collins RD. Immunologic characterization of human malignant lymphomas. Cancer 1974;34:1488-03.

${ }^{3}$ Lennert K. Malignant lymphomas other than Hodgkin's disease: histology, cytology, ultrastructure, immunology. Heidelberg: Springer Verlag, 1978.

${ }^{4}$ National Cancer Institute. Sponsored study of classifications of non-Hodgkin's lymphomas. Summary of description of a working formulation for clinical usage. Cancer 1982;49:2112-35.

${ }^{5}$ Leonard RCF, Cuzick J. Maclennan ICM, et al. Prognostic factors in non-Hodgkin's lymphomas: the importance of symptomatic stage as an adjunct to the Kiel histopathological classification. Br J Cancer 1983;47:91-02.

${ }^{6}$ Molenaar WM, Bartels H, Koudstaal J. Histological, epidemiological and clinical aspects of centroblastic-centrocytic lymphomas subdivided according to the "working formulation". Br J Cancer 1984;49:263-68.

${ }^{7}$ Herrman R, Barcos M, Stutzman L, et al. The influence of histologic type on the incidence and duration of response in nonHodgkin's lymphoma. Cancer 1982;49:314.

${ }^{8}$ Cooper EH, Peckham MJ, Millard RE, Hamlin IME, GerardMarchant R. Cell proliferation in human malignant lymphomas. Analysis of labelling index and DNA content in cell populations obtained by biopsy. Eur J Cancer 1968;4:287-96.

${ }^{9}$ Simonsson B, Nilsson K. ${ }^{3} \mathrm{H}$-Thymidine uptake in chronic lymphatic leukaemia cells. Scand J Haematol 1977;24:169-73.

${ }^{10}$ Silvestrini R, Piazza R, Riccardi A, Rilke F. Correlation of cell kinetic findings with morphology of non-Hodgkin's malignant lymphomas. JNCI 1977;58:499-04.

${ }^{11}$ Kvaloy S, Godal T, Marton PF, Steen H, Brennhoyd IO, Abrahamsen AF. Spontaneous $\left({ }^{3} \mathbf{H}\right)$-Thymidine uptake in histological subgroups of human B-cell lymphomas. Scand J Haematol 1981;26:221-34.
${ }^{12}$ Costa A, Bonadonna G, Villa E, Valagussa P, Silvestrini R. Labelling index as a prognostic marker in non-Hodgkin's lymphoma. JNCI 1981;66:1-5.

${ }^{13}$ Braylan RC, Fowlkes BJ, Jaffe ES, Sanders SK, Berard CW, Herman CJ. Cell volumes and DNA distributions of normal and neoplastic human lymphoid cells. Cancer 1978;41:201-09.

${ }^{14}$ Shackney SE, Skramstad KS, Cunningham RE, Dugas DJ, Lincoln TL, Lukes RJ. Dual parameter flow cytometry studies in human lymphomas. J Clin Invest 1980;66:1281-94.

15 Diamond LW, Braylan RC. Flow analysis of DNA content and cell size in non-Hodgkin's lymphomas. Cancer Res 1980; 40:703-12.

${ }^{16}$ Barlogie B, Raber MN, Schumann J, et al. Flow cytometry in clinical cancer research. Cancer Res 1983;43:3982.

${ }^{17}$ Diamond LW, Nathwani BN, Rappaport H. Flow cytometry in the diagnosis and classification of malignant lymphoma and leukemia. Cancer 1982;50:1122-35.

${ }^{18}$ Braylan RC, Benson NA, Nourse VA. Cellular DNA of human neoplastic B-cells measured by flow cytometry. Cancer Res 1984;44:5010-16.

${ }^{19}$ Habeshaw JA, Lister TA, Stansfeld AG, Greaves MF. Correlation of transferrin receptor expression with histological class and outcome in non-Hodgkin's lymphoma. Lancet 1983;i:498-01.

${ }^{20}$ Gerdes J, Dallenbach F, Lennert K, Lemke H, Stein H. Growth fractions in malignant non-Hodgkin's lymphomas (NHL) as determined in situ with the monoclonal antibody $\mathrm{Ki}-67$. Haematological Oncology 1984;2:365-71.

${ }^{21}$ Costa A, Mazzini G, Delbino G, Silvestrini R. DNA content and proliferative characteristics of non-Hodgkin's lymphomas determined by flow cytometry and autoradiography. Cytometry 1981;2:185-88

${ }^{22}$ Braylan RC, Diamond LW, Powell ML, Harty-Golder B. Percentage of cells in the S-phase of the cell cycle in human lymphoma determined by flow cytometry: correlation with labelling index and survival. Cytometry 1980;1:171-74.

${ }^{23}$ Camplejohn RS, Macartney JC. Comparison of DNA flow cytometry from fresh and paraffin embedded samples of nonHodgkin's lymphoma. J Clin Pathol 1985;38:1096-99.

${ }^{24}$ Oviatt DL, Cousar JB, Collins RD, Flexner JM, Stein RS. Malignant lymphomas of follicular center cell origin in humans $\mathrm{V}$. Incidence, clinical features, and prognostic implications of transformation of small cleaved cell nodular lymphomas. Cancer 1984;53:1109-14.

${ }^{25}$ Hedley DW, Friedlander ML, Taylor IW, Rugg CA, Musgrove EA. Method for analysis of cellular DNA content of paraffin-embedded pathological material using flow cytometry. J Histochem Cytochem 1983;31:1333-5.

${ }^{26}$ Baisch H, Gohde W, Linden WA. Analysis of PCP-data to determine the fraction of cells in the various phases of cell cycle. Radiat Environ Biophys 1975;12:31-9.

${ }^{27}$ Frankfurt OS, Greco WR, Slocum HK, et al. Proliferative characteristics of primary and metastitic human solid tumors by DNA flow cytometry. Cytometry 1984;5:629-35.

${ }^{28}$ Wright N, Alison M. The biology of epithelial cell populations. Vol 1. Oxford: Clarendon Press, 1984.

Requests for reprints to: Dr JC Macartney, Department of Histopathology, North Wing Ward Block, St Thomas's Hospital, London SE1 7EH, England. 